PROCEEDINGS OF THE

AMERICAN MATHEMATICAL SOCIETY

Volume 129, Number 12, Pages 3501-3510

S 0002-9939(01)06273-6

Article electronically published on June 27, 2001

\title{
INVARIANT SUBSPACES AND REPRESENTATIONS OF CERTAIN VON NEUMANN ALGEBRAS
}

\author{
TOMOYOSHI OHWADA, GUOXING JI, AND KICHI-SUKE SAITO
}

(Communicated by David R. Larson)

\begin{abstract}
Let $(N, \alpha, G)$ be a covariant system and let $(\pi, U)$ be a covariant representation of $(N, \alpha, G)$ on a Hilbert space $\mathcal{H}$. In this note, we investigate the representation of the covariance algebra $M$ and the $\sigma$-weakly closed subalgebra $\mathfrak{A}$ generated by $\pi(N)$ and $\left\{U_{g}\right\}_{g \geq 0}$ in the case of $G=\mathbb{Z}$ or $\mathbb{R}$ when there exists a pure, full, $\mathfrak{A}$-invariant subspace of $\mathcal{H}$.
\end{abstract}

\section{INTRODUCTION}

If $G$ is a locally compact group and $\alpha: G \rightarrow \operatorname{Aut}(N)$ is a continuous homomorphism of $G$ into the group of *-automorphisms of a von Neumann algebra $N$, then the triple $(N, \alpha, G)$ is called a covariant system. This notation was introduced by Doplicher, Kastler and Robinson in 2]. Covariant systems have turned out to be very interesting objects, both in theoretical physics and in mathematics. The covariant representation of $(N, \alpha, G)$ means a pair $(\pi, U)$ consisting of a unitary representation $U$ of $G$ and a *-representation $\pi$ of $N$ with $\pi$ and $U$ operating over the same Hilbert space $\mathcal{H}$ such that

$$
\pi\left(\alpha_{g}(x)\right)=U_{g} \pi(x) U_{g}^{*} \quad(\forall x \in N, \forall g \in G) .
$$

The covariance algebra $M$ of $(N, \alpha, G)$ is a von Neumann algebra generated by $\pi(N)$ and $\left\{U_{g}\right\}_{g \in G}$. When $G$ has an order $\geq$, we consider the $\sigma$-weakly closed subalgebra $\mathfrak{A}$ of $M$ which is generated by $\pi(N)$ and $\left\{U_{g}\right\}_{g \geq 0}$. The representation theory of $M$ has been extensively studied by M. Takesaki in [16], M. Landstad in [5] and I. Raeburn in [13, among others. The covariance algebras provide us with a rich variety of examples of operator algebras. In this note, we consider the representation theory of $M$ and $\mathfrak{A}$ in the particular case of $G=\mathbb{R}$ or $\mathbb{Z}$. By inspiring the scattering theory of Lax and Phillips in [6], we study the representation of $M$ and $\mathfrak{A}$ to a crossed product and an analytic crossed product, respectively, using the theory of invariant subspace for $\mathfrak{A}$. Therefore, our setting is as follows.

Let $M$ be a von Neumann algebra acting on a Hilbert space $\mathcal{H}$ generated by a von Neumann algebra $N$ and a unitary operator $v$ satisfying $v N v^{*}=N$, and let $\mathfrak{A}$ be a $\sigma$-weakly closed subalgebra of $M$ generated by $N$ and the non-negative powers of $v$. At first, we prove that if there is a pure, full, $\mathfrak{A}$-invariant subspace $\mathfrak{M}$

Received by the editors September 16, 1999.

2000 Mathematics Subject Classification. Primary 46L10, 47L65; Secondary 46L40.

This work was supported in part by a Grant-in-Aid for Scientific Research, Japan Society for Promotion of Science. 
of $\mathcal{H}$, then $M$ is $*$-isomorphic to a (discrete) crossed product $N \rtimes_{\alpha} \mathbb{Z}$ of $N$ by a *-automorphism $\alpha=\operatorname{ad} v$, and that $\mathfrak{A}$ is simultaneously isomorphic to the analytic crossed product $N \rtimes_{\alpha} \mathbb{Z}_{+}$.

Similarly, we also consider the representation of a von Neumann algebra $M_{0}$ generated by a von Neumann algebra $N$ and a strongly continuous one-parameter unitary group $\left\{u_{t}\right\}_{t \in \mathbb{R}}$ satisfying $u_{t} N u_{t}^{*}=N$ for every $t$ in $\mathbb{R}$. Let $\mathfrak{B}$ be the $\sigma$ weakly closed subalgebra of $M_{0}$ generated by $N$ and $\left\{u_{t}\right\}_{t>0}$. We prove that if there is a pure, full, $\mathfrak{B}$-invariant subspace of $\mathcal{H}$, then $M_{0}$ is $*$-isomorphic to a continuous crossed product, and that $\mathfrak{B}$ is simultaneously isomorphic to the related analytic crossed product.

Next, in $\S 3$, for a strongly continuous one-parameter unitary group $\left\{u_{t}\right\}_{t \in \mathbb{R}}$, we construct the unitary operator $v$ by the Cayley transform of the infinitesimal generator of $\left\{u_{t}\right\}_{t \in \mathbb{R}}$. From this unitary $v$ and $\left\{u_{t}\right\}_{t \in \mathbb{R}}$, we define the von Neumann algebras $M, M_{0}$ and the subalgebras $\mathfrak{A}, \mathfrak{B}$, respectively, as in $\S 2$, and we shall show that a closed subspace of $\mathcal{H}$ is pure, full, $\mathfrak{A}$-invariant if and only if it is pure, full, $\mathfrak{B}$-invariant (Proposition 3.2). Finally, we shall discuss the relation between a discrete crossed product and continuous crossed product.

\section{Representation of Certain von Neumann algebras TO A CROSSED PRODUCT}

At first, we consider the representation of the covariance subalgebra in the case of $G=\mathbb{Z}$. Let $M$ be a von Neumann algebra acting on a Hilbert space $\mathcal{H}$ generated by a von Neumann algebra $N$ and a unitary operator $v$ satisfying $v N v^{*}=N$ and let $\mathfrak{A}$ be the $\sigma$-weakly closed subalgebra of $M$ generated by $N$ and non-negative powers of $v$. We now define the notion of invariant subspaces of $\mathcal{H}$ with respect to $\mathfrak{A}$ as in [8]-10].

Definition 2.1. Let $\mathfrak{M}$ be a closed subspace of $\mathcal{H}$. We shall say that $\mathfrak{M}$ is: $\mathfrak{A}$ invariant, if $\mathfrak{A M} \subset \mathfrak{M}$; reducing, if $M \mathfrak{M} \subset \mathfrak{M}$; pure, if $\mathfrak{M}$ contains no non-trivial reducing subspace; and full, if the smallest reducing subspace containing $\mathfrak{M}$ is all of $\mathcal{H}$.

Since $v N v^{*}=N$, we put $\alpha(x)=v x v^{*}(\forall x \in N)$. We recall that the crossed product $N \rtimes_{\alpha} \mathbb{Z}$ of $N$ by the $*$-automorphism group $\left\{\alpha^{n}\right\}_{n \in \mathbb{Z}}$ is the von Neumann algebra acting on the Hilbert space $\ell^{2}(\mathbb{Z}, \mathcal{H})$ generated by the operators $\pi_{\alpha}(x)(\forall x \in$ $N)$ and $S$ defined by the equations

$$
\left\{\pi_{\alpha}(x) \xi\right\}(n)=\alpha^{-n}(x) \xi(n) \quad\left(\forall \xi \in \ell^{2}(\mathbb{Z}, \mathcal{H}), \forall n \in Z\right)
$$

and

$$
(S \xi)(n)=\xi(n-1) \quad\left(\forall \xi \in \ell^{2}(\mathbb{Z}, \mathcal{H}), \forall n \in \mathbb{Z}\right) .
$$

We note that the analytic crossed product $N \rtimes_{\alpha} \mathbb{Z}_{+}$determined by $N$ and $\alpha$ is defined to be the $\sigma$-weakly closed subalgebra of $N \rtimes_{\alpha} \mathbb{Z}$ generated by $\pi_{\alpha}(N)$ and the non-negative powers of $S$ (cf. 8]-[10]). Let $\left\{\widehat{\alpha}_{t}\right\}_{t \in \mathbb{T}}$ be the $*$-automorphism group of $N \rtimes_{\alpha} \mathbb{Z}$ which is dual to $\left\{\alpha^{n}\right\}_{n \in \mathbb{Z}}$ in the sense of Takesaki [16]. Then we have

Theorem 2.2. Let $M$ be a von Neumann algebra acting on a Hilbert space $\mathcal{H}$ generated by a von Neumann algebra $N$ and a unitary operator $v$ satisfying $v N v^{*}=$ $N$ and let $\mathfrak{A}$ be the $\sigma$-weakly closed subalgebra of $M$ generated by $N$ and non-negative powers of $v$. Put $\alpha(x)=v x v^{*}(\forall x \in N)$. If there exists a pure, full, $\mathfrak{A}$-invariant 
subspace $\mathfrak{M}$ of $\mathcal{H}$, then there exist a $*$-automorphism group $\left\{\gamma_{t}\right\}_{t \in \mathbb{T}}$ of $M$ and a *-isomorphism $\Phi$ from $M$ onto $N \rtimes_{\alpha} \mathbb{Z}$ such that

$$
\Phi(x)=\pi_{\alpha}(x)(\forall x \in N), \quad \Phi(v)=S \quad \text { and } \quad \Phi \circ \gamma_{t}=\widehat{\alpha_{t}} \circ \Phi(\forall t \in \mathbb{T}) .
$$

Proof. Let $\mathfrak{M}$ be a pure, full, $\mathfrak{A}$-invariant subspace of $\mathcal{H}$. As in [8, Proposition 3.1], the subspace $\mathfrak{M}$ has the following properties:

$$
\text { (i) } \mathfrak{A M} \subset \mathfrak{M}, \quad \text { (ii) } \bigcap_{k>0} v^{k} \mathfrak{M}=\{0\}, \quad \text { (iii) } \overline{\bigcup_{k<0} v^{k} \mathfrak{M}}=\mathcal{H} .
$$

Putting $\mathfrak{F}=\mathfrak{M} \ominus v \mathfrak{M}$, we have the decomposition of the Hilbert space $\mathcal{H}$ :

$$
\mathcal{H}=\sum_{n=-\infty}^{\infty} \oplus v^{n} \mathfrak{F}
$$

Let $P_{n}$ be the projection from $\mathcal{H}$ onto $v^{n} \mathfrak{F}$. Since $\mathfrak{F}$ is $N$-invariant and $v N v^{*}=N$, it follows that $P_{n}$ belongs to the commutant of $N$ and $\sum_{n=-\infty}^{\infty} P_{n}=I$. We define the one-parameter unitary group $\left\{V_{t}\right\}_{t \in \mathbb{T}}$ in the commutant of $N$ defined by

$$
V_{t}=\sum_{n=-\infty}^{\infty} e^{i n t} P_{n} \quad(\forall t \in \mathbb{T}) .
$$

For each $t \in \mathbb{T}$, we see that

$$
\begin{aligned}
v V_{t} v^{*} & =\sum_{n=-\infty}^{\infty} e^{i n t} v P_{n} v^{*}=\sum_{n=-\infty}^{\infty} e^{i n t} P_{n+1} \\
& =\sum_{n=-\infty}^{\infty} e^{i(n-1) t} P_{n}=e^{-i t} \sum_{n=-\infty}^{\infty} e^{i n t} P_{n} \\
& =e^{-i t} V_{t} .
\end{aligned}
$$

Setting $\gamma_{t}(x)=V_{t}^{*} x V_{t}$ for each $t \in \mathbb{T}$ and $x \in M$, we see that $\left\{\gamma_{t}\right\}_{t \in \mathbb{T}}$ is a $*-$ automorphism group of $M$ such that $\gamma_{t}(v)=e^{-i t} v(\forall t \in \mathbb{T})$. By [15, 19.9 Theorem], we have this proposition.

We now fix a pure, full, $\mathfrak{A}$-invariant subspace $\mathfrak{M}$ of $\mathcal{H}$. Then, by Theorem 2.2, there exist a $*$-automorphism group $\left\{\gamma_{t}\right\}_{t \in \mathbb{T}}$ of $M$ and a $*$-isomorphism $\Phi$ from $M$ onto $N \rtimes_{\alpha} \mathbb{Z}$ such that

$$
\Phi(x)=\pi_{\alpha}(x)(\forall x \in N), \quad \Phi(v)=S \quad \text { and } \quad \Phi \circ \gamma_{t}=\widehat{\alpha_{t}} \circ \Phi(\forall t \in \mathbb{T}) .
$$

On the other hand, we take another pure, full, $\mathfrak{A}$-invariant subspace $\mathfrak{N}$ of $\mathcal{H}$. As in the proof of Theorem 2.2, there exists a unitary group $\left\{W_{t}\right\}_{t \in \mathbb{T}}$ in the commutant of $N$ associated with $\mathfrak{N}$. Put $\rho_{t}(x)=W_{t} x W_{t}^{*}(\forall x \in M)$. By Theorem 2.2, there exists a $*$-isomorphism $\Psi$ from $M$ onto $N \rtimes_{\alpha} \mathbb{Z}$ such that

$$
\Psi(x)=\pi_{\alpha}(x)(\forall x \in N), \quad \Psi(v)=S \quad \text { and } \quad \Psi \circ \rho_{t}=\widehat{\alpha_{t}} \circ \Psi(\forall t \in \mathbb{T}) .
$$

Therefore, we have

$$
\Phi \circ \gamma_{t} \circ \Phi^{-1}=\widehat{\alpha_{t}}=\Psi \circ \rho_{t} \circ \Psi^{-1} \quad(\forall t \in \mathbb{T}) .
$$

Since $\Phi^{-1} \circ \Psi$ is the identity map on $M$, we see that $\gamma_{t}=\rho_{t}(\forall t \in \mathbb{T})$ and so $V_{t} x V_{t}^{*}=W_{t} x W_{t}^{*}(\forall x \in M, \forall t \in \mathbb{T})$. Putting $A_{t}=W_{t}^{*} V_{t}(\forall t \in \mathbb{T})$, then $A_{t}$ is the unitary operator in the commutant of $M$ and, for all $s, t \in \mathbb{T}$, we have

$$
A_{t} V_{t}^{*} A_{s} V_{t}=W_{t}^{*} V_{t} V_{t}^{*} W_{s}^{*} V_{s} V_{t}=W_{t+s}^{*} V_{t+s}=A_{t+s} .
$$


Thus, we have

$$
A_{t+s}=A_{t} \gamma_{-t}\left(A_{s}\right) \quad(\forall s, t \in \mathbb{T}) .
$$

The unitary family $\left\{A_{t}\right\}_{t \in \mathbb{T}}$ in the commutant of $M$ satisfying (2.1) is called a cocycle with respect to $\mathfrak{M}$. Therefore we have

Theorem 2.3. Let $M$ be a von Neumann algebra acting on a Hilbert space $\mathcal{H}$ generated by a von Neumann algebra $N$ and a unitary operator $v$ satisfying $v N v^{*}=$ $N$ and let $\mathfrak{A}$ be the $\sigma$-weakly closed subalgebra of $M$ generated by $N$ and non-negative powers of $v$. Let $\mathfrak{M}$ be a pure, full, $\mathfrak{A}$-invariant subspace of $\mathcal{H}$. If $\mathfrak{N}$ is another pure, full, $\mathfrak{A}$-invariant subspace of $\mathcal{H}$, then there exists a cocycle $\left\{A_{t}\right\}_{t \in \mathbb{T}}$ with respect to $\mathfrak{M}$. Conversely, if $\left\{A_{t}\right\}_{t \in \mathbb{T}}$ is a cocycle with respect to $\mathfrak{M}$, then there exists a pure full $\mathfrak{A}$-invariant subspace of $\mathcal{H}$ with the cocycle $\left\{A_{t}\right\}_{t \in \mathbb{T}}$.

Proof. We only prove the converse. Assume that $\left\{A_{t}\right\}_{t \in \mathbb{T}}$ is a cocycle with respect to $\mathfrak{M}$. Put $W_{t}=V_{t}^{*} A_{t}(\forall t \in \mathbb{T})$. Then we can easily check that $\left\{W_{t}\right\}_{t \in \mathbb{T}}$ is a unitary group in the commutant of $N$. Let $W_{t}=\sum_{n=-\infty}^{\infty} e^{-i n t} Q_{n}(\forall t \in \mathbb{T})$ be the spectral decomposition of $W_{t}$. Putting $\mathfrak{N}=\sum_{n=0}^{\infty} \oplus Q_{n} \mathcal{H}$, then $\mathfrak{N}$ is a pure, full, $\mathfrak{A}-$ invariant subspace of $\mathcal{H}$. In fact, for each $x \in N$ and $\xi \in \mathcal{H}$, we have $x Q_{n} \xi \in Q_{n} \mathcal{H}$ because $W_{t}$ and $A_{t}$ belong to $N^{\prime}$. Moreover, we see that

$$
\begin{aligned}
W_{t} v Q_{n} \xi= & V_{t}^{*} A_{t} v Q_{n} \xi=V_{t}^{*} v A_{t} Q_{n} \xi=e^{-i t} v V_{t}^{*} A_{t} Q_{n} \xi \\
& =e^{-i t} v W_{t} Q_{n} \xi=e^{-i(n+1) t} v Q_{n} \xi .
\end{aligned}
$$

It follows that $v Q_{n} \xi \in Q_{n+1} \mathcal{H}(\forall n \in \mathbb{N})$. Therefore $\mathfrak{N}$ is $\mathfrak{A}$-invariant. This completes the proof.

We next consider the case that $G=\mathbb{R}$. Let $N$ be a von Neumann algebra acting on a Hilbert space $\mathcal{H}$ and let $\left\{u_{t}\right\}_{t \in \mathbb{R}}$ be a strongly continuous one-parameter unitary group on $\mathcal{H}$ satisfying the condition $u_{t} N u_{t}{ }^{*}=N(\forall t \in \mathbb{R})$. Let $M_{0}$ be the von Neumann algebra generated by $N$ and $\left\{u_{t}\right\}_{t \in \mathbb{R}}$, and let $\mathfrak{B}$ be the $\sigma$-weakly closed subalgebra of $M_{0}$ generated by $N$ and $\left\{u_{t}\right\}_{t \geq 0}$.

Definition 2.4. Let $\mathfrak{M}$ be a closed subspace of $\mathcal{H}$. We shall say that $\mathfrak{M}$ is: $\mathfrak{B}$ invariant, if $\mathfrak{B M} \subset \mathfrak{M}$; reducing, if $M_{0} \mathfrak{M} \subset \mathfrak{M}$; pure, if $\mathfrak{M}$ contains no non-trivial reducing subspace; and full, if the smallest reducing subspace containing $\mathfrak{M}$ is all of $\mathcal{H}$.

Since $u_{t}$ and $N$ satisfy the condition $u_{t} N u_{t}{ }^{*}=N(\forall t \in \mathbb{R})$, we can define the $\sigma$-weakly continuous $*$-automorphism $\beta_{t}$ of $N$ implemented by the unitary operator $u_{t}(\forall t \in \mathbb{R})$. Recall that the continuous crossed product $N \rtimes_{\beta} \mathbb{R}$ of $N$ by $\left\{\beta_{t}\right\}_{t \in \mathbb{R}}$ is the von Neumann algebra acting on a Hilbert space $L^{2}(\mathbb{R}, \mathcal{H})$ generated by the operators $\pi_{\beta}(x)$ and $\lambda(t)$ defined by the equations, for $\forall x \in N$,

$$
\left\{\pi_{\beta}(x) \xi\right\}(t)=\beta_{-t}(x) \xi(t) \quad\left(\forall \xi \in L^{2}(\mathbb{R}, \mathcal{H}), \forall t \in \mathbb{R}\right)
$$

and

$$
\{\lambda(t) \xi\}(s)=\xi(s-t) \quad\left(\forall \xi \in L^{2}(\mathbb{R}, \mathcal{H}), \forall s, t \in \mathbb{R}\right) .
$$

The analytic crossed product $N \rtimes_{\beta} \mathbb{R}_{+}$determined by $N$ and $\left\{\beta_{t}\right\}_{t \in \mathbb{R}}$ is defined to be the $\sigma$-weakly closed subalgebra of $N \rtimes_{\beta} \mathbb{R}$ generated by $\pi_{\beta}(N)$ and $\{\lambda(t)\}_{t \geq 0}$. 
Theorem 2.5. Let $M_{0}$ be the von Neumann algebra generated by $N$ and a unitary group $\left\{u_{t}\right\}_{t \in \mathbb{R}}$ satisfying $u_{t} N u_{t}^{*}=N(\forall t \in \mathbb{R})$ and let $\mathfrak{B}$ be the $\sigma$-weakly closed subalgebra of $M_{0}$ generated by $N$ and $\left\{u_{t}\right\}_{t \geq 0}$. If there exists a pure, full, $\mathfrak{B}-$ invariant subspace $\mathfrak{M}$ of $\mathcal{H}$, then there exist a one-parameter group $\left\{\theta_{t}\right\}_{t \in \mathbb{R}}$ of $*-$ automorphisms on $M_{0}$ and a*-isomorphism $\Theta$ from $M_{0}$ onto $N \rtimes_{\beta} \mathbb{R}$ such that

$$
\Theta(x)=\pi_{\beta}(x)(\forall x \in N), \quad \Theta\left(u_{t}\right)=\lambda(t) \quad \text { and } \quad \Theta \circ \theta_{t}=\widehat{\beta}_{t} \circ \Theta(\forall t \in \mathbb{R}),
$$

where $\left\{\widehat{\beta}_{t}\right\}_{t \in \mathbb{R}}$ is the *-automorphism of $N \rtimes_{\beta} \mathbb{R}$ which is dual to $\left\{\beta_{t}\right\}_{t \in \mathbb{R}}$. Further $\Theta$ maps $\mathfrak{A}$ onto $N \rtimes_{\beta} \mathbb{R}_{+}$.

Proof. Let $\mathfrak{M}$ be a pure, full, $\mathfrak{B}$-invariant subspace of $\mathcal{H}$. Then it is clear that $\mathfrak{M}$ has the following properties:

$$
\text { (i) } \mathfrak{B M} \subset \mathfrak{M}, \quad \text { (ii) } \bigcap_{t>0} u_{t} \mathfrak{M}=\{0\}, \quad \text { (iii) } \overline{\bigcup_{t<0} u_{t} \mathfrak{M}}=\mathcal{H}
$$

Let $P_{t}$ be the projection from $\mathcal{H}$ onto $u_{t} \mathfrak{M}(\forall t \in \mathbb{R})$. Since $N \mathfrak{M} \subset \mathfrak{M}$ and $N=$ $u_{t} N u_{t}^{*}$, for each $t \in \mathbb{R}, u_{t} \mathfrak{M}$ is $N$-invariant, and so $P_{t}$ belongs to $N^{\prime}$. Since $\mathfrak{M}$ is pure and full, we can easily check that the projections $\left\{P_{t}\right\}_{t \in \mathbb{R}}$ are a spectral family. Thus, we obtain the strongly continuous unitary group $\left\{U_{t}\right\}_{t \in \mathbb{R}}$ of $N^{\prime}$ defined by

$$
U_{t}=\int_{\mathbb{R}} e^{-i \lambda t} d P_{\lambda} \quad(\forall t \in \mathbb{R}) .
$$

Since $u_{s}^{*} P_{\lambda} u_{s}=u_{\lambda-s}$, we have for every $s, t \in \mathbb{R}$,

$$
\begin{aligned}
u_{s}^{*} U_{t} u_{s} & =\int_{\mathbb{R}} e^{-i \lambda t} d u_{s}^{*} P_{\lambda} u_{s}=\int_{\mathbb{R}} e^{-i \lambda t} d P_{\lambda-s} \\
& =e^{-i s t} \int_{\mathbb{R}} e^{-i \lambda t} d P_{\lambda}=e^{-i s t} U_{t} .
\end{aligned}
$$

Therefore $U_{t} x U_{t}^{*}=x(\forall x \in N \forall t \in \mathbb{R})$ and $U_{t} u_{s} U_{t}^{*}=e^{-i s t} u_{s}(\forall s, t \in \mathbb{R})$. Thus the *-automorphism group $\left\{\theta_{t}\right\}_{t \in \mathbb{R}}$ of $M_{0}$ defined by $\theta_{t}(x)=U_{t} x U_{t}^{*}\left(\forall x \in M_{0}, \forall t \in \mathbb{R}\right)$ satisfies $\theta_{t}\left(u_{s}\right)=e^{-i s t} u_{s}(\forall s, t \in \mathbb{R})$. Therefore we have the proposition from [15] 19.9 Theorem].

We now fix a pure, full, $\mathfrak{B}$-invariant subspace $\mathfrak{M}$ of $\mathcal{H}$. As in the case of $G=\mathbb{Z}$, we can consider the notion of cocycle with respect to $\mathfrak{M}$. By Theorem 2.5, there exist a one-parameter group $\left\{\theta_{t}\right\}_{t \in \mathbb{R}}$ of $*$-automorphisms on $M_{0}$ which is implemented by the unitary group $\left\{U_{t}\right\}_{t \in \mathbb{T}}$ and a $*$-isomorphism $\Theta$ from $M_{0}$ onto $N \rtimes_{\beta} \mathbb{R}$ such that

$$
\Theta(x)=\pi_{\beta}(x)(\forall x \in N), \quad \Theta\left(u_{t}\right)=\lambda(t) \quad \text { and } \quad \Theta \circ \theta_{t}=\widehat{\beta}_{t} \circ \Theta(\forall t \in \mathbb{R}) .
$$

We take another pure, full, $\mathfrak{B}$-invariant subspace $\mathfrak{N}$ of $\mathcal{H}$. As in the proof of Theorem 2.5, there exists a one-parameter unitary group $\left\{W_{t}\right\}_{t \in \mathbb{R}}$ associated with $\mathfrak{N}$. Put $\sigma_{t}(x)=W_{t} x W_{t}^{*}$ for any $x \in M_{0}$. Then, by Theorem 2.5 , there exists a *-isomorphism $\Pi$ from $M_{0}$ onto $N \rtimes_{\beta} \mathbb{R}$ such that

$$
\Pi(x)=\pi_{\beta}(x)(\forall x \in N), \quad \Pi\left(u_{t}\right)=\lambda(t) \quad \text { and } \quad \Pi \circ \sigma_{t}=\widehat{\beta}_{t} \circ \Pi(\forall t \in \mathbb{R}) .
$$

Put $B_{t}=W_{t}^{*} U_{t}(\forall t \in \mathbb{R})$. Then $B_{t}$ is a unitary operator in the commutant of $M_{0}$ satisfying $B_{t+s}=B_{t} \theta_{-t}\left(B_{s}\right)(\forall s, t \in \mathbb{R})$. We shall say that the unitary family $\left\{B_{t}\right\}_{t \in \mathbb{R}}$ is a cocycle with respect to $\mathfrak{M}$. As in Theorem 2.3, we have the following: 
Theorem 2.6. Let $M_{0}$ be the von Neumann algebra generated by $N$ and a unitary group $\left\{u_{t}\right\}_{t \in \mathbb{R}}$ satisfying $u_{t} N u_{t}^{*}=N(\forall t \in \mathbb{R})$ and let $\mathfrak{B}$ be the $\sigma$-weakly closed subalgebra of $M_{0}$ generated by $N$ and $\left\{u_{t}\right\}_{t \geq 0}$. Let $\mathfrak{M}$ be a pure, full, $\mathfrak{B}$-invariant subspace of $\mathcal{H}$. If we take another pure, full, $\mathfrak{B}$-invariant subspace $\mathfrak{N}$ of $\mathcal{H}$, then there exists a cocycle $\left\{B_{t}\right\}_{t \in \mathbb{R}}$ with respect to $\mathfrak{M}$. Conversely, if $\left\{B_{t}\right\}_{t \in \mathbb{R}}$ is a cocycle with respect to $\mathfrak{M}$, then there exists a pure, full, $\mathfrak{B}$-invariant subspace of $\mathcal{H}$ with the cocycle $\left\{B_{t}\right\}_{t \in \mathbb{R}}$.

\section{Representation of the continuous case $G=\mathbb{R}$}

Let $N$ be a von Neumann algebra acting on a Hilbert space $\mathcal{H}$ and let $\left\{u_{t}\right\}_{t \in \mathbb{R}}$ be a strongly continuous one-parameter unitary group on $\mathcal{H}$. We consider the von Neumann algebra $M_{0}$ generated by $N$ and $\left\{u_{t}\right\}_{t \in \mathbb{R}}$, and the $\sigma$-weakly closed subalgebra $\mathfrak{B}$ of $M_{0}$ generated by $N$ and $\left\{u_{t}\right\}_{t \geq 0}$. Let $A$ be the infinitesimal generator of $\left\{u_{t}\right\}_{t \in \mathbb{R}}$ defined by

$$
A \xi=\lim _{t \rightarrow 0+} \frac{u_{t} \xi-\xi}{t} \quad(\forall \xi \in D(A))
$$

where $D(A)$ is the set of all elements for which the limit exists. It is well-known that the Cayley transform $v$ of $A$, that is, $v=(I+A)(I-A)^{-1}$, is a unitary operator on $\mathcal{H}$. For the unitary operator $v$, let $M$ be the von Neumann algebra generated by $N$ and $v$, and let $\mathfrak{A}$ be the $\sigma$-weakly closed subalgebra generated by $N$ and the non-negative powers of $v$ as in $\S 2$.

The next proposition embodies an important idea of $[\underline{6}]$ and is the key result of our approach. For completeness, we give the proof.

Proposition 3.1. Keep the notations as above. Let $\mathfrak{M}$ be a closed subspace of $\mathcal{H}$. Then $\mathfrak{M}$ is $\mathfrak{A}$-invariant if and only if $\mathfrak{M}$ is $\mathfrak{B}$-invariant.

Proof. We only need to prove that a closed subspace $\mathfrak{M}$ of $\mathcal{H}$ is $v$-invariant if and only if $\mathfrak{M}$ is $u_{t}$-invariant for all $t>0$. Let $A$ be a infinitesimal generator of $\left\{u_{t}\right\}_{t \in \mathbb{R}}$. Setting $R(\lambda, A)=(\lambda I-A)^{-1}$, we see that

$$
v=R(1, A)+A R(1, A)=2 R(1, A)-I,
$$

and making use of the Laplace transform representation of $R(1, A)$, we have

$$
v \xi=2 \int_{0}^{\infty} e^{-t} u_{t} \xi d t-\xi \quad \forall \xi \in \mathcal{H}
$$

Let $\xi \in \mathfrak{M}$. Since $u_{t} \xi \in \mathfrak{M}$ for all $t>0$, we have $v \xi \mathfrak{M}$.

To prove the converse, we first show that $R(\lambda, A) \mathfrak{M} \subset \mathfrak{M}$ for all $\lambda>0$. Now the resolvent is analytic on the resolvent set and can be expanded in a power series as follows:

$$
R(\lambda, A)=\sum_{n=0}^{\infty}\left(\lambda_{0}-\lambda\right)^{n}\left\{R\left(\lambda_{0}, A\right)\right\}^{n+1},
$$

valid for $\left|\lambda_{0}-\lambda\right|\left|R\left(\lambda_{0}, A\right)\right|<1$. For $\lambda_{0}>0$, we have $\left|R\left(\lambda_{0}, A\right)\right| \leqq \frac{1}{\lambda_{0}}$ so that the above series holds for $\left|\lambda-\lambda_{0}\right|<\lambda_{0}$. It follows from this expansion that $R\left(\lambda_{0}, A\right) \mathfrak{M} \subset$ $\mathfrak{M}$ implies $R(\lambda, A) \mathfrak{M} \subset \mathfrak{M}$ for all $\left|\lambda-\lambda_{0}\right|<\lambda_{0}$. Assuming $v \mathfrak{M} \subset \mathfrak{M}$, one infers from (3.1) that $R(1, A) \mathfrak{M} \subset \mathfrak{M}$ and hence by a stepwise process using (3.3) that 
$R(\lambda, A) \mathfrak{M} \subset \mathfrak{M}$ for all $\lambda>0$. Hence for $\xi$ in $\mathfrak{M}$ and $\eta$ in the orthogonal complement of $\mathfrak{M}$

$$
0=\langle R(\lambda, A) \xi, \eta\rangle=\int_{0}^{\infty} e^{-\lambda t}\left\langle u_{t} \xi, \eta\right\rangle d t \quad(\forall \lambda>0) .
$$

By the Laplace transform uniqueness theorem, we have $\left\langle u_{t} \xi, \eta\right\rangle=0$ and hence $u_{t} \mathfrak{M} \subset \mathfrak{M}$ for all $t>0$. This completes the proof.

From Proposition 3.1, we have the following:

Proposition 3.2. Keep the notation as above. Then

(i) $M=M_{0}$. Moreover, if $M_{0}$ has a separating vector, then $\mathfrak{A}=\mathfrak{B}$.

(ii) A closed subspace $\mathfrak{M}$ of $\mathcal{H}$ is pure, full, $\mathfrak{A}$-invariant if and only if $\mathfrak{M}$ is pure, full, $\mathfrak{B}$-invariant.

Proof. We only prove (i). By Proposition 3.1, a closed subspace $\mathfrak{M}$ is reducing for $M$ if and only if $\mathfrak{M}$ is reducing for $M_{0}$. Hence the commutant of $M$ is equal to the commutant of $M_{0}$, and so $M=M_{0}$.

We next prove that $\mathfrak{A}=\mathfrak{B}$. To do this, we need the following notations. If $\mathfrak{C}$ is an algebra of $M_{0}$ and $\mathfrak{L}$ is a lattice of projections in $B(\mathcal{H})$, then we write

$$
\text { Lat } \mathfrak{C}=\left\{P \in B(\mathcal{H})_{p} \mid(I-P) T P=0, \forall T \in \mathfrak{C}\right\}
$$

and

$$
\operatorname{Alg} \mathfrak{L}=\{T \in B(\mathcal{H}) \mid(I-P) T P=0, \forall P \in \mathfrak{L}\},
$$

where $B(\mathcal{H})_{p}$ is the set of all projections in $B(\mathcal{H})$.

By Proposition 3.1, it is clear that Lat $\mathfrak{A}=$ Lat $\mathfrak{B}$. Since AlgLat $\mathfrak{B}$ contains $\mathfrak{B}$, we have the following inclusions:

$$
\mathfrak{A} \subset \mathfrak{B} \subset \text { AlgLat } \mathfrak{A} .
$$

If $\mathfrak{A} \varsubsetneqq$ AlgLatA, then there exists a non-zero element $x \in \operatorname{AlgLat} \mathfrak{A}$ such that $x \notin \mathfrak{A}$. Hence there is a normal linear functional $\phi$ in the predual $\left(M_{0}\right)_{*}$ of $M_{0}$ such that $\phi(x)=1$ and $\left.\phi\right|_{\mathfrak{A}}=0$. Since $M_{0}$ has a separating vector, by [7] Corollary 1.13.7], there are non-zero vectors $\xi$ and $\eta$ in $\mathcal{H}$ such that $\phi(y)=\langle y \xi, \eta\rangle(\forall y \in M)$. Hence, for each $y \in \mathfrak{A}$, we have

$$
\langle y \xi, \eta\rangle=\phi(y)=0 .
$$

This implies that $[\mathfrak{A} \xi] \perp \eta$, where $[\mathfrak{A} \xi]$ denotes the closed subspace of $\mathcal{H}$ spanned by $\mathfrak{A} \xi$. Since $[\mathfrak{A} \xi] \in \operatorname{Lat} \mathfrak{A}$ and $x \in \operatorname{AlgLat} \mathfrak{A}, x \xi$ belongs to $[\mathfrak{A} \xi]$. This implies that

$$
1=\phi(x)=\langle x \xi, \eta\rangle=0 .
$$

This is a contradiction and so $\mathfrak{A}=\mathfrak{B}$. This completes the proof.

We remark that if the commutant of a von Neumann algebra $M_{0}$ is properly infinite, then $M_{0}$ always has a separating vector (cf. [1, Corollary 11]).

We now consider the case that $N$ and $u_{t}$ satisfy the condition $u_{t} N u_{t}^{*}=N(\forall t \in$ $\mathbb{R})$. If there exists a pure, full, $\mathfrak{B}$-invariant subspace $\mathfrak{M}$ of $\mathcal{H}$, then, by Theorem 2.5, $M_{0}$ is $*$-isomorphic to a continuous crossed product. Moreover, from 
Proposition 3.2(ii), $\mathfrak{M}$ is also a pure, full, $\mathfrak{A}$-invariant subspace. Hence, by Theorem 2.2, if $N$ and $v$ satisfy the condition $v N v^{*}=N$, then $M$ is $*$-isomorphic to a discrete crossed product. So it is natural to ask when $N$ and $v$ satisfy the condition $v N v^{*}=N$. Recall that the unitary operator $v$ has the following form:

$$
v \xi=2 \int_{0}^{\infty} e^{-t} u_{t} \xi d t-\xi \quad(\forall \xi \in \mathcal{H})
$$

It is clear that if, for every $t \in \mathbb{R}, u_{t}$ belongs to the commutant of $N$, then $v$ is also in $N^{\prime}$. In this case, $v$ and $N$ satisfy the condition $v N v^{*}=N$. But, in general, $v$ and $N$ do not satisfy the condition $v N v^{*}=N$. In fact, we can give the following:

Example 3.3. Let $N$ be a von Neumann algebra acting on a Hilbert space $\mathcal{H}$ and let $\left\{\beta_{t}\right\}_{t \in \mathbb{R}}$ be a $*$-automorphism group of $N$ such that there exists a $t_{0}$ in $\mathbb{R}$ such that $\beta_{t_{0}}$ is outer. Recall that a continuous crossed product $N \rtimes_{\beta} \mathbb{R}$ is the von

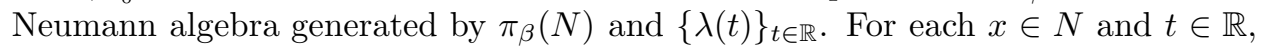
it is clear that

$$
\pi_{\beta}\left(\beta_{t}(x)\right)=\lambda(t) \pi_{\beta}(x) \lambda(t)^{*}
$$

and

$$
\pi_{\beta}(N)=\lambda(t) \pi_{\beta}(N) \lambda(t)^{*} \quad(\forall t \in \mathbb{R}) .
$$

For the unitary group $\{\lambda(t)\}_{t \in \mathbb{R}}$, we obtain the unitary operator $v$ defined by the form

$$
v \xi=2 \int_{0}^{\infty} e^{-t} \lambda(t) \xi d t-\xi \quad\left(\forall \xi \in L^{2}(\mathbb{R}, \mathcal{H})\right)
$$

By choosing an appropriate representation for $N \rtimes_{\beta} \mathbb{R}$, we shall assume that $N \rtimes_{\beta}$ $\mathbb{R}$ has a separating vector. In this case, by Proposition 3.2 , the von Neumann algebra generated by $\pi_{\beta}(N)$ and $v$ coincides with $N \rtimes_{\beta} \mathbb{R}$, and the $\sigma$-weakly closed subalgebra generated by $\pi_{\beta}(N)$ and the non-negative powers of $v$ also coincides with $N \rtimes_{\beta} \mathbb{R}_{+}$. Hence, by Theorem 2.2 , if $\pi_{\beta}(N)$ and $v$ satisfy the condition $v \pi_{\beta}(N) v^{*}=\pi_{\beta}(N)$, then there is a $*$-isomorphism $\Phi$ from $N \rtimes_{\beta} \mathbb{R}$ onto $N \rtimes_{\alpha} \mathbb{Z}$ such that $\Phi\left(N \rtimes_{\beta} \mathbb{R}_{+}\right)=N \rtimes_{\alpha} \mathbb{Z}_{+}$for some $*$-automorphism $\alpha$ of $N$. Since there exists a faithful normal canonical conditional expectation of $N \rtimes_{\alpha} \mathbb{Z}$ onto $\pi_{\alpha}(N)$ (cf. [8] [10]), there is a faithful normal conditional expectation of $N \rtimes_{\beta} \mathbb{R}$ onto $\pi_{\beta}(N)$. However, Katayama showed in [4. Theorem 3.5] that if there exists a $t_{0}$ in $\mathbb{R}$ such that $\beta_{t_{0}}$ is outer, then there does not exist any normal conditional expectation of $N \rtimes_{\beta} \mathbb{R}$ onto $\pi_{\beta}(N)$, which is a contradiction. Hence, $\pi_{\beta}(N)$ and $v$ do not satisfy the condition $v \pi_{\beta}(N) v^{*}=\pi_{\beta}(N)$.

Finally, we discuss the relation between a continuous crossed product and a discrete crossed product.

Theorem 3.4. If a crossed product $N \rtimes_{\beta} \mathbb{R}$ admits a separating vector (for example, $N \rtimes_{\beta} \mathbb{R}$ is properly infinite), then the following two conditions are equivalent:

(i) There exists a *-isomorphism $\Phi$ form $N \rtimes_{\beta} \mathbb{R}$ onto $N \rtimes_{\alpha} \mathbb{Z}$ such that $\Phi\left(N \rtimes_{\beta} \mathbb{R}_{+}\right)=N \rtimes_{\alpha} \mathbb{Z}_{+}$for some $*$-automorphism $\alpha$ of $N$.

(ii) $\beta_{t}$ is inner for all $t \in \mathbb{R}$.

Proof. (i) $\Rightarrow$ (ii) Since $\Phi$ is the $*$-isomorphism satisfying $\Phi\left(N \rtimes_{\beta} \mathbb{R}_{+}\right)=N \rtimes_{\alpha} \mathbb{Z}_{+}$, we have $\Phi\left(\pi_{\beta}(N)\right)=\pi_{\alpha}(N)$. Since there exists a normal conditional expectation of $N \rtimes_{\alpha} \mathbb{Z}$ onto $\pi_{\alpha}(N)$, there also exists a normal conditional expectation of $N \rtimes_{\beta} \mathbb{R}$ onto $\pi_{\beta}(N)$. Hence, by [4, Theorem 3.6], we have that $\beta_{t}$ is inner for each $t \in \mathbb{R}$. 
(ii) $\Rightarrow$ (i) Since $\beta_{t}$ is inner for all $t \in \mathbb{R}$, there exists a unitary operator $v_{t}$ in $N$ such that $\beta_{t}$ is implemented by the unitary operator $v_{t}$. Putting $u_{t}=\lambda(t) \pi_{\beta}\left(v_{t}\right)^{*}$ for all $t$ in $\mathbb{R}$, we can show that $\left\{u_{t}\right\}_{t \in \mathbb{R}}$ is a strongly continuous one-parameter unitary group in $\pi_{\beta}(N)^{\prime}$. Moreover, we see that the von Neumann algebra generated by $\pi_{\beta}(N)$ and $\left\{u_{t}\right\}_{t \in \mathbb{R}}$ is equal to $N \rtimes_{\beta} \mathbb{R}$. Similarly, the $\sigma$-weakly closed subalgebra of $N \rtimes_{\beta} \mathbb{R}$ generated by $\pi_{\beta}(N)$ and $\left\{u_{t}\right\}_{t>0}$ is $N \rtimes_{\beta} \mathbb{R}_{+}$. For the unitary group $\left\{u_{t}\right\}_{t \in \mathbb{R}}$, we can construct the unitary operator $v$ on $L^{2}(\mathbb{R}, \mathcal{H})$ as follows:

$$
v \xi=2 \int_{0}^{\infty} e^{-t} u_{t} \xi d t-\xi \quad\left(\forall \xi \in L^{2}(\mathbb{R}, \mathcal{H})\right)
$$

Since $N \rtimes_{\beta} \mathbb{R}$ admits a separating vector, by Proposition $3.2, N \rtimes_{\beta} \mathbb{R}$ is also generated by $\pi_{\beta}(N)$ and $v$, and $N \rtimes_{\beta} \mathbb{R}_{+}$is generated by $\pi_{\beta}(N)$ and the nonnegative powers of $v$. Since, for every $t \in \mathbb{R}, u_{t}$ belongs to the commutant of $\pi_{\beta}(N)$, $v$ is also in $\pi_{\beta}(N)^{\prime}$. Thus $v$ and $\pi_{\beta}(N)$ satisfy the condition $v \pi_{\beta}(N) v^{*}=\pi_{\beta}(N)$. Putting $\mathfrak{M}=L^{2}\left(\mathbb{R}_{+}, \mathcal{H}\right)$, it is clear that $\mathfrak{M}$ is a pure, full, $N \rtimes_{\beta} \mathbb{R}_{+}$-invariant subspace of $L^{2}(\mathbb{R}, \mathcal{H})$. For each $t \in \mathbb{R}$, we have

$$
u_{t} \mathfrak{M}=\lambda(t) \pi_{\beta}\left(v_{t}\right)^{*} \mathfrak{M} \subset \lambda(t) \mathfrak{M}=\pi_{\beta}\left(v_{t}\right)^{*} \lambda(t) \pi_{\beta}\left(v_{t}\right) \mathfrak{M} \subset u_{t} \mathfrak{M}
$$

It follows that $u_{t} \mathfrak{M}=\lambda(t) \mathfrak{M}(\forall t \in \mathbb{R})$, and so $\mathfrak{M}$ is pure and full for $\left\{u_{t}\right\}_{t \in \mathbb{R}}$. Therefore, by Proposition 3.2, $\mathfrak{M}$ is also pure and full for $v$. Thus, by Theorem 2.2, we have (i). This completes the proof.

\section{REFERENCES}

1. J. Dixmier, Von Neumann algebras, North-Holland, Amsterdam, 1981. MR 83a:46004

2. D. Doplicher, D. Kastler and D. Robinson, Covariance algebras in field theory and statistical mechanics, Comm. Math. Phys., 3 (1966), 1-28. MR 34:4930

3. R. V. Kadison and J. R. Ringrose, Fundamentals of the theory of operator algebras I, Academic Press, 1983. MR 85j:46099

4. Y. Katayama, Non-existence of a normal conditional expectation in a continuous crossed product, Kodai Math. J., 4 (1981), 345-352. MR 83a:46078

5. M. Landstad, Duality theory for covariant systems, Trans. Amer. Math. Soc., 248 (1979), 223-267. MR 80j:46107

6. P. Lax and R. Phillips, Scattering Theory, 2nd ed., Academic Press, New York, 1989. MR 90k:35005

7. B. R. Li, Introduction to operator algebras, World Scientific Publ., 1992. MR 94b:46083

8. M. McAsey, P. S. Muhly and K-S. Saito, Non-selfadjoint crossed products (Invariant subspaces and maximality), Trans. Amer. Math. Soc., 248 (1979), 381-409. MR 80j:46101b

9. M. McAsey, P. S. Muhly and K-S. Saito, Non-selfadjoint crossed products II, J. Math. Soc. Japan, 33 (1981), 485-495. MR 83a:46071

10. M. McAsey, P. S. Muhly and K-S. Saito, Non-selfadjoint crossed products III, J. Operator Theory, 12 (1984), 3-22. MR 86d:46057

11. P. S. Muhly and K-S. Saito, Analytic crossed products and outer conjugacy classes of automorphisms of von Neumann algebras, Math. Scand., 58 (1986), 55-68. MR 87i:46137

12. P. S. Muhly and K-S. Saito, Analytic crossed products and outer conjugacy classes of automorphisms of von Neumann algebras II, Math. Ann., 279 (1987), 1-7. MR 89a:46137

13. I. Raeburn, On crossed products and Takai duality, Proc. Edinburgh Math. Soc. 31 (1988), 321-330. MR 90d:46093

14. K-S. Saito, Invariant subspaces and cocycles in nonselfadjoint crossed products, J. Funct. Anal., 45 (1982), 177-193. MR 84a:46141 Corrigendum MR 86c:46075 
15. S. Strătilaă, Modular theory in operator algebras, Abacus Press, 1981. MR 85g:46072

16. M. Takesaki, Duality for crossed products and the structure of von Neumann algebras of type III, Acta Math., 131 (1973), 249-310. MR 55:11068

Department of Mathematics, General Education, Tsuruoka National College of Technology, TSuruoka, 997-8511, JaPAN

E-mail address: ohwada@tsuruoka-nct.ac.jp

Department of Mathematics, Shaanxi Normal University, Xian, 710062, Shaanxi, PeoPLE'S Republic of China

E-mail address: gxji@dns.snnu.edu.cn

Department of Mathematics, Faculty of Science, Nitgata University, Niigata, 950-21, JAPAN

E-mail address: saito@math.sc.niigata-u.ac.jp 